\title{
INTEGRAL AND LIMIT REPRESENTATIONS OF THE CMP INVERSE
}

\author{
DIJANA MOSIĆ \\ Received 29 December, 2019
}

\begin{abstract}
We develop various integral and limit representations for the CMP inverse of a complex square matrix, which do not require any restriction on the spectrum of a corresponding matrix. Also, we present integral and limit representations for the DMP and MPD inverses.
\end{abstract}

2010 Mathematics Subject Classification: 15A09; 65F20

Keywords: CMP inverse, Drazin inverse, Moore-Penrose inverse, DMP inverse

\section{INTRODUCTION}

Let $\mathbb{C}^{m \times n}$ be the set of all $m \times n$ complex matrices. We use $\operatorname{rank}(A), A^{*}, R(A)$ and $N(A)$ to denote the rank, the conjugate transpose, the range (column space) and the null space of $A \in \mathbb{C}^{m \times n}$, respectively. The index of $A \in \mathbb{C}^{n \times n}$, denoted by $\operatorname{ind}(A)$, is the smallest nonnegative integer $k$ for which $\operatorname{rank}\left(A^{k}\right)=\operatorname{rank}\left(A^{k+1}\right)$. By $I$ will be denoted the identity matrix of corresponding size. If $M$ and $N$ are two complementary subspaces of $\mathbb{C}^{m \times 1}$ (that is, $\mathbb{C}^{m \times 1}$ is direct sum of $M$ and $N$ ), we denote by $P_{M, N}$ the projector onto $M$ along $N$. In the case that $N$ is the subspace orthogonal to $M$, this notation will be reduced to $P_{M}$.

The Drazin inverse of $A \in \mathbb{C}^{n \times n}$ is the unique matrix $A^{D}=X \in \mathbb{C}^{n \times n}$ such that

$$
A^{k+1} X=A^{k}, \quad X A X=X, \quad A X=X A .
$$

where $k=\operatorname{ind}(A)$. If $\operatorname{ind}(A)=1$, then $A^{D}$ is the group inverse of $A$, which is denoted by $A^{\#}$. For basic properties of the Drazin inverse and its various applications see $[1,3]$.

The Moore-Penrose inverse of $A \in \mathbb{C}^{m \times n}$ is the unique matrix $A^{\dagger}=X \in \mathbb{C}^{n \times m}$ which satisfies the Penrose equations

$$
A X A=A, \quad X A X=X, \quad(A X)^{*}=A X, \quad(X A)^{*}=X A .
$$

The first author was supported by the Ministry of Education and Science, Republic of Serbia, Grant No. 174007 (451-03-68/2020-14). 
The Moore-Penrose inverse is a powerful tool in computing polar decomposition, the areas of electrical networks, control theory, filtering, estimation theory and pattern recognition.

Let $A \in \mathbb{C}^{m \times n}$ be of rank $r$, let $T$ be a subspace of $\mathbb{C}^{n}$ of dimension $s \leq r$, and let $S$ be a subspace of $\mathbb{C}^{m}$ of dimension $m-s$. If a matrix $X \in \mathbb{C}^{n \times m}$ satisfies

$$
X A X=X, \quad R(X)=T, \quad N(X)=S,
$$

then $X$ is called the outer inverse of $A$ with the range $T$ and the null-space $S$, and the notation $X=A_{T, S}^{(2)}$ is commonly used. Drazin [6] introduced a new class of outer inverses, called the $(B, C)$-inverses. For $A \in \mathbb{C}^{m \times n}$ and $B, C \in \mathbb{C}^{n \times m}$, if a matrix $X \in$ $\mathbb{C}^{n \times m}$ satisfies $X A B=B, C A X=C, R(X) \subseteq R(B)$ and $N(C) \subseteq N(X)$, then $X$ is called the $(B, C)$-inverse of $A$. In the case when $X$ exists, it is unique and denoted by $X=$ $A^{\|(B, C)}[2,6]$. By [2, Theorem 7.1], it follows that $A^{\|(B, C)}=A_{R(B), N(C)}^{(2)}$.

Using the Drazin inverse and the Moore-Penrose inverse, Malik and Thome [9] defined a new generalized inverse of a square matrix of an arbitrary index, which is called the DMP inverse and defined as $A^{D, \dagger}=A^{D} A A^{\dagger}$, for $A \in \mathbb{C}^{n \times n}$. The DMP inverse for a Hilbert space operator was investigated in $[13,17,19]$ as a generalization of the DMP inverse for a square matrix. For $A \in \mathbb{C}^{n \times n}$, the MPD inverse, as the dual DMP inverse, was given by $A^{\dagger, D}=A^{\dagger} A A^{D}$ [9].

Mehdipour and Salemi [10] introduced a new inverse of a square matrix $A$ named CMP inverse, since they used the core part $A A^{D} A$ of $A$ and the Moore-Penrose inverse of $A$. The CMP inverse of $A \in \mathbb{C}^{n \times n}$ is defined as $A^{c, \dagger}=A^{\dagger} A A^{D} A A^{\dagger}$ and it is the unique solution of the following equations:

$$
X A X=X, \quad A X A=A A^{D} A, \quad A X=A A^{D} A A^{\dagger}, \quad X A=A^{\dagger} A A^{D} A .
$$

For more details about the CMP inverse see $[12,18]$.

It is well-known that if the eigenvalues of $A \in \mathbb{C}^{n \times n}$ lie in the open right halfplane, then the inverse of $A$ can be presented by

$$
A^{-1}=\int_{0}^{\infty} \exp (-t A) d t
$$

Many integral representations of various generalized inverse such as Moore-Penrose inverse, Drazin inverse and DMP inverse were presented in papers [4,5,7,20]. Several of these integral representations have some restriction on the eigenvalues of $A$ and the other holds without any restrictions on the eigenvalues.

Notice that investigation of the limit representations of different kinds of generalized inverses are hot topics many years. One limit representation of the Drazin inverse was proved by Meyer [11] in 1974. Some limit representations of the outer inverse are given in $[8,15,16]$.

The above mentioned results motivate us to investigate the integral and limit representations of the CMP inverse of a square matrix, without any restriction on the spectrum of a certain matrix. Firstly, we develop these representations based on 
the full-rank decomposition of a given matrix. Then we establish integral and limit representations of the CMP inverse which depend on corresponding projections and expressions for the Moore-Penrose, Drazin and outer inverses. Various integral and limit representations of the DMP and MPD inverses are also derived.

\section{INTEGRAL REPRESENTATIONS OF THE CMP INVERSE}

In this section, we will establish integral representations of the CMP inverse for a square complex matrix without any restriction on the spectrum of matrix. If $A \in \mathbb{C}^{n \times n}$ is nilpotent, then $A^{D}=0$ and so $A^{c, \dagger}=0$. Since this case is trivial, we consider the matrix $A$ to be non-nilpotent in this paper.

Lemma 1 ([1]). Let $A \in \mathbb{C}^{n \times n}$ with $\operatorname{ind}(A)=k$. If $A=B_{1} G_{1}$ is a full-rank decomposition and $G_{i} B_{i}=B_{i+1} G_{i+1}$ are also full-rank decompositions, $i=1,2, \ldots, k-1$. Then the following statements hold:

(i) $G_{k} B_{k}$ is invertible;

(ii) $A^{k}=B_{1} B_{2} \ldots B_{k} G_{k} \ldots G_{2} G_{1}$;

(iii) $A^{D}=B_{1} B_{2} \ldots B_{k}\left(G_{k} B_{k}\right)^{-k-1} G_{k} \ldots G_{2} G_{1}$;

(iv) $A^{\dagger}=G_{1}^{*}\left(G_{1} G_{1}^{*}\right)^{-1}\left(B_{1}^{*} B_{1}\right)^{-1} B_{1}^{*}$.

In particular, for $k=1$, then $G_{1} B_{1}$ is invertible and $A^{\#}=B_{1}\left(G_{1} B_{1}\right)^{-2} G_{1}$.

Lemma 2. Let $A \in \mathbb{C}^{n \times n}$ with $\operatorname{ind}(A)=k$ and the full-rank decomposition of $A$ as in Lemma 1. Then

$$
G_{1} A^{c, \dagger} B_{1}=B_{2} \ldots B_{k}\left(G_{k} B_{k}\right)^{-(k-1)} G_{k} \ldots G_{2} .
$$

Proof. By [20, Lemma 3.1], we have that $A^{D, \dagger} B_{1}=B_{1} B_{2} \ldots B_{k}\left(G_{k} B_{k}\right)^{-k} G_{k} \ldots G_{2}$ which implies

$$
A^{c, \dagger} B_{1}=A^{\dagger} A A^{D, \dagger} B_{1}=A^{\dagger} A B_{1} \ldots B_{k}\left(G_{k} B_{k}\right)^{-k} G_{k} \ldots G_{2} .
$$

Therefore, by Lemma 1 ,

$$
\begin{aligned}
G_{1} A^{c, \dagger} B_{1} & =G_{1} A^{\dagger} A B_{1} \ldots B_{k}\left(G_{k} B_{k}\right)^{-k} G_{k} \ldots G_{2} \\
& =G_{1} G_{1}^{*}\left(G_{1} G_{1}^{*}\right)^{-1}\left(B_{1}^{*} B_{1}\right)^{-1} B_{1}^{*} B_{1} G_{1} B_{1} \ldots B_{k}\left(G_{k} B_{k}\right)^{-k} G_{k} \ldots G_{2} \\
& =G_{1} B_{1} \ldots B_{k}\left(G_{k} B_{k}\right)^{-k} G_{k} \ldots G_{2}=B_{2} G_{2} B_{2} \ldots B_{k}\left(G_{k} B_{k}\right)^{-k} G_{k} \ldots G_{2} \\
& =B_{2} \ldots G_{k} B_{k}\left(G_{k} B_{k}\right)^{-k} G_{k} \ldots G_{2}=B_{2} \ldots B_{k}\left(G_{k} B_{k}\right)^{-(k-1)} G_{k} \ldots G_{2} .
\end{aligned}
$$

Theorem 1. Let $A \in \mathbb{C}^{n \times n}$ with $\operatorname{ind}(A)=k$ and the full-rank decomposition of $A$ as in Lemma 1. Then

$$
A^{c, \dagger}=\int_{0}^{\infty} G_{1}^{*} \exp \left(-G_{1} G_{1}^{*} t\right) d t \int_{0}^{\infty} M B_{1}^{*} \exp \left(-B_{1} B_{1}^{*} u\right) d u,
$$

where $M=B_{2} \ldots B_{k}\left(G_{k} B_{k}\right)^{-(k-1)} G_{k} \ldots G_{2}$. 
Proof. Set $X=G_{1}^{\dagger} M B_{1}^{\dagger}$. Recall that, by [7],

$$
A^{\dagger}=\int_{0}^{\infty} A^{*} \exp \left(-A A^{*} t\right) d t
$$

It is enough to prove that $X=A^{c, \dagger}$. Because $B_{1}$ is a full-column rank matrix, then $B_{1}^{\dagger}=\left(B_{1}^{*} B_{1}\right)^{-1} B_{1}^{*}$ and so $B_{1}^{\dagger} B_{1}=I$. Similarly, we have that $G_{1}^{\dagger}=G_{1}^{*}\left(G_{1} G_{1}^{*}\right)^{-1}$ and $G_{1} G_{1}^{\dagger}=I$. Notice that, using

$$
G_{k} \ldots G_{2} B_{2} \ldots B_{k}=G_{k} \ldots G_{3} B_{3} G_{3} B_{3} \ldots B_{k}=\cdots=\left(G_{k} B_{k}\right)^{k-1},
$$

we get

$$
\begin{aligned}
X A X & =G_{1}^{\dagger} B_{2} \ldots B_{k}\left(G_{k} B_{k}\right)^{-(k-1)} G_{k} \ldots G_{2} B_{1}^{\dagger} B_{1} G_{1} G_{1}^{\dagger} M B_{1}^{\dagger} \\
& =G_{1}^{\dagger} B_{2} \ldots B_{k}\left(G_{k} B_{k}\right)^{-(k-1)} G_{k} \ldots G_{2} M B_{1}^{\dagger} \\
& =G_{1}^{\dagger} B_{2} \ldots B_{k}\left(G_{k} B_{k}\right)^{-(k-1)} G_{k} \ldots G_{2} B_{2} \ldots B_{k}\left(G_{k} B_{k}\right)^{-(k-1)} G_{k} \ldots G_{2} B_{1}^{\dagger} \\
& =G_{1}^{\dagger} B_{2} \ldots B_{k}\left(G_{k} B_{k}\right)^{-(k-1)}\left(G_{k} B_{k}\right)^{k-1}\left(G_{k} B_{k}\right)^{-(k-1)} G_{k} \ldots G_{2} B_{1}^{\dagger} \\
& =G_{1}^{\dagger} B_{2} \ldots B_{k}\left(G_{k} B_{k}\right)^{-(k-1)} G_{k} \ldots G_{2} B_{1}^{\dagger} \\
& =X .
\end{aligned}
$$

Applying Lemma 1, we observe that

$$
\begin{aligned}
A A^{D} A & =B_{1} G_{1} B_{1} B_{2} \ldots B_{k}\left(G_{k} B_{k}\right)^{-k-1} G_{k} \ldots G_{2} G_{1} B_{1} G_{1} \\
& =B_{1} B_{2} G_{2} B_{2} \ldots B_{k}\left(G_{k} B_{k}\right)^{-k-1} G_{k} \ldots G_{2} B_{2} G_{2} G_{1} \\
& =B_{1} B_{2} \ldots B_{k} G_{k} B_{k}\left(G_{k} B_{k}\right)^{-k-1} G_{k} B_{k} G_{k} \ldots G_{2} G_{1} \\
& =B_{1} B_{2} \ldots B_{k}\left(G_{k} B_{k}\right)^{-(k-1)} G_{k} \ldots G_{2} G_{1} .
\end{aligned}
$$

Therefore,

$$
\begin{aligned}
X A & =G_{1}^{\dagger} B_{2} \ldots B_{k}\left(G_{k} B_{k}\right)^{-(k-1)} G_{k} \ldots G_{2} G_{1} \\
& =G_{1}^{*}\left(G_{1} G_{1}^{*}\right)^{-1}\left(B_{1}^{*} B_{1}\right)^{-1} B_{1}^{*} B_{1} B_{2} \ldots B_{k}\left(G_{k} B_{k}\right)^{-(k-1)} G_{k} \ldots G_{2} G_{1} \\
& =A^{\dagger} A A^{D} A
\end{aligned}
$$

and

$$
\begin{aligned}
A X & =B_{1} B_{2} \ldots B_{k}\left(G_{k} B_{k}\right)^{-(k-1)} G_{k} \ldots G_{2} B_{1}^{\dagger} \\
& =B_{1} B_{2} \ldots B_{k}\left(G_{k} B_{k}\right)^{-(k-1)} G_{k} \ldots G_{2} G_{1} G_{1}^{*}\left(G_{1} G_{1}^{*}\right)^{-1}\left(B_{1}^{*} B_{1}\right)^{-1} B_{1}^{*} \\
& =A A^{D} A A^{\dagger} .
\end{aligned}
$$

By [12, Corollary 2.2], we deduce that $X=A^{c, \dagger}$.

Notice that we represent the CMP inverse by two integrals in Theorem 1. In order to simplify integral representation of the CMP inverse, we firstly use the DMP inverse, MPD inverse and orthogonal projections. 
Theorem 2. Let $A \in \mathbb{C}^{n \times n}$ with $\operatorname{ind}(A)=k$ and the full-rank decomposition of $A$ as in Lemma 1. Then

$$
A^{c, \dagger}=\int_{0}^{\infty} P_{R\left(A^{*}\right)} M_{1} B_{1}^{*} \exp \left(-B_{1} B_{1}^{*} u\right) d u=\int_{0}^{\infty} G_{1}^{*} \exp \left(-G_{1} G_{1}^{*} t\right) M_{2} P_{R(A)} d t,
$$

where $M_{1}=B_{1} B_{2} \ldots B_{k}\left(G_{k} B_{k}\right)^{-(k-1)} G_{k} \ldots G_{2}$ and $M_{2}=B_{2} \ldots B_{k}\left(G_{k} B_{k}\right)^{-(k-1)} G_{k} \ldots$ $G_{2} G_{1}$.

Proof. Based on $A^{c, \dagger}=P_{R\left(A^{*}\right)} A^{D, \dagger}=A^{\dagger, D} P_{R(A)}$ and [20, Theorem 3.2], we obtain this result.

Applying an integral representation for the Drazin inverse showed in [4], which does not require any restriction on its eigenvalues, we give the following integral representations for the CMP inverse.

Theorem 3. Let $A \in \mathbb{C}^{n \times n}$ with $\operatorname{ind}(A)=k$. Then

$$
A^{c, \dagger}=\int_{0}^{\infty} P_{R\left(A^{*}\right)} \exp \left[-t A^{k}\left(A^{2 k+1}\right)^{*} A^{k+1}\right] A^{k}\left(A^{2 k+1}\right)^{*} A^{k} P_{R(A)} d t .
$$

Proof. It follows by the equality $A^{c, \dagger}=P_{R\left(A^{*}\right)} A^{D} P_{R(A)}$ and the next integral representation for the Drazin inverse proved in [4, Theorem 2.1]:

$$
A^{D}=\int_{0}^{\infty} \exp \left[-t A^{k}\left(A^{2 k+1}\right)^{*} A^{k+1}\right] A^{k}\left(A^{2 k+1}\right)^{*} A^{k} d t .
$$

As Theorem 3, new integral representations for the DMP and MPD inverses are obtained.

Corollary 1. Let $A \in \mathbb{C}^{n \times n}$ with $\operatorname{ind}(A)=k$. Then

$$
A^{D, \dagger}=\int_{0}^{\infty} P_{R\left(A^{*}\right)} \exp \left[-t A^{k}\left(A^{2 k+1}\right)^{*} A^{k+1}\right] A^{k}\left(A^{2 k+1}\right)^{*} A^{k} d t
$$

and

$$
A^{\dagger, D}=\int_{0}^{\infty} \exp \left[-t A^{k}\left(A^{2 k+1}\right)^{*} A^{k+1}\right] A^{k}\left(A^{2 k+1}\right)^{*} A^{k} P_{R(A)} d t .
$$

We present more expressions for the CMP inverse involving one integral.

Theorem 4. Let $A \in \mathbb{C}^{n \times n}$ with ind $(A)=k$. Then

$$
A^{c, \dagger}=\int_{0}^{\infty} A^{*} \exp \left(-A A^{*} t\right) P_{R\left(A^{k}\right), N\left(A^{k}\right)} P_{R(A)} d t=\int_{0}^{\infty} P_{R\left(A^{*}\right)} P_{R\left(A^{k}\right), N\left(A^{k}\right)} A^{*} \exp \left(-A A^{*} t\right) d t .
$$

Proof. The equalities $A^{c, \dagger}=A^{\dagger} P_{R\left(A^{k}\right), N\left(A^{k}\right)} P_{R(A)}=P_{R\left(A^{*}\right)} P_{R\left(A^{k}\right), N\left(A^{k}\right)} A^{\dagger}$ and (2.1) yield these formulae.

Similarly as Theorem 4, we show some formulae for the DMP inverse and MPD inverse. 
Corollary 2. Let $A \in \mathbb{C}^{n \times n}$ with $\operatorname{ind}(A)=k$. Then

$$
A^{D, \dagger}=\int_{0}^{\infty} A^{*} \exp \left(-A A^{*} t\right) P_{R\left(A^{k}\right), N\left(A^{k}\right)} d t
$$

and

$$
A^{\dagger, D}=\int_{0}^{\infty} P_{R\left(A^{*}\right)} P_{R\left(A^{k}\right), N\left(A^{k}\right)} A^{*} \exp \left(-A A^{*} t\right) d t .
$$

Theorem 5. Let $A \in \mathbb{C}^{n \times n}$ with $\operatorname{ind}(A)=k$. If $G \in \mathbb{C}^{n \times n}$ such that $R(G)=R\left(A^{\dagger} A^{k}\right)$ and $N(G)=N\left(A^{k} A^{\dagger}\right)$, then

$$
A^{c, \dagger}=\int_{0}^{\infty} \exp \left[-G(G A G)^{*} G A t\right] G(G A G)^{*} G d t .
$$

Proof. Using [14, Corollary 3.7], we have $A^{c, \dagger}=A_{R\left(A^{\dagger} A^{D}\right), N\left(A^{D} A^{\dagger}\right)}^{(2)}=A_{R\left(A^{\dagger} A^{k}\right), N\left(A^{k} A^{\dagger}\right)}^{(2)}$. By [17, Theorem 2.2] (or [2, Corollary 7.6]), we obtain

$$
A_{R\left(A^{\dagger} A^{k}\right), N\left(A^{k} A^{\dagger}\right)}^{(2)}=\int_{0}^{\infty} \exp \left[-G(G A G)^{*} G A t\right] G(G A G)^{*} G d t .
$$

Using the integral representation for the $(B, C)$-inverse proved in [2], we obtain the next integral representation for the CMP inverse based on some restriction on the eigenvalues of corresponding matrix.

Theorem 6. Let $A \in \mathbb{C}^{n \times n}$ with ind $(A)=k$ and let $G \in \mathbb{C}^{n \times n}$ such that $R(G)=$ $R\left(A^{\dagger} A^{k}\right)$ and $N(G)=N\left(A^{k} A^{\dagger}\right)$. If the nonzero spectrum of $G A$ lies in the open left half plane, then

$$
A^{c, \dagger}=-\int_{0}^{\infty} \exp (G A t) G d t
$$

Proof. It follows by $A^{c, \dagger}=A_{R\left(A^{\dagger} A^{k}\right), N\left(A^{k} A^{\dagger}\right)}^{(2)}=A^{\|\left(A^{\dagger} A^{k}, A^{k} A^{\dagger}\right)}$ and [2, Corollary 7.7].

\section{LIMIT REPRESENTATIONS OF THE CMP INVERSE}

In the beginning of this section, we present the limit representation of the CMP inverse based on the full-rank decomposition of $A$ given in Lemma 1.

Theorem 7. Let $A \in \mathbb{C}^{n \times n}$ with $\operatorname{ind}(A)=k$ and the full-rank decomposition of $A$ as in Lemma 1. Then

$$
A^{c, \dagger}=\lim _{\lambda \rightarrow 0} G_{1}^{*}\left(\lambda I+G_{1} G_{1}^{*}\right)^{-1} \lim _{t \rightarrow 0} M\left(t I+B_{1}^{*} B_{1}\right)^{-1} B_{1}^{*},
$$

where $M=B_{2} \ldots B_{k}\left(G_{k} B_{k}\right)^{-(k-1)} G_{k} \ldots G_{2}$. 
Proof. We have, by [15],

$$
A^{\dagger}=\lim _{\lambda \rightarrow 0} A^{*}\left(\lambda I+A A^{*}\right)^{-1}=\lim _{\lambda \rightarrow 0}\left(\lambda I+A^{*} A\right)^{-1} A^{*} .
$$

For $X=G_{1}^{\dagger} M B_{1}^{\dagger}$, we check that $X=A^{c, \dagger}$ as in the proof of Theorem 1 .

To avoid two limits, we included orthogonal projections in limit representations of CMP inverse. Similarly as Theorem 7 and Theorem 2, we verify the following result.

Theorem 8. Let $A \in \mathbb{C}^{n \times n}$ with $\operatorname{ind}(A)=k$ and the full-rank decomposition of $A$ as in Lemma 1. Then

$$
A^{c, \dagger}=\lim _{\lambda \rightarrow 0} P_{R\left(A^{*}\right)} M_{1} B_{1}^{*}\left(\lambda I+B_{1} B_{1}^{*}\right)^{-1}=\lim _{\lambda \rightarrow 0} G_{1}^{*}\left(\lambda I+G_{1} G_{1}^{*}\right)^{-1} M_{2} P_{R(A)} d t,
$$

where $M_{1}=B_{1} B_{2} \ldots B_{k}\left(G_{k} B_{k}\right)^{-(k-1)} G_{k} \ldots G_{2}$ and $M_{2}=B_{2} \ldots B_{k}\left(G_{k} B_{k}\right)^{-(k-1)} G_{k} \ldots$ $G_{2} G_{1}$.

Analogously, we can prove the limit representations of DMP and MPD inverses.

Corollary 3. Let $A \in \mathbb{C}^{n \times n}$ with $\operatorname{ind}(A)=k$ and the full-rank decomposition of $A$ as in Lemma 1. Then

$$
A^{D, \dagger}=\lim _{\lambda \rightarrow 0} M_{1} B_{1}^{*}\left(\lambda I+B_{1} B_{1}^{*}\right)^{-1}
$$

and

$$
A^{\dagger, D}=\lim _{\lambda \rightarrow 0} G_{1}^{*}\left(\lambda I+G_{1} G_{1}^{*}\right)^{-1} M_{2} d t,
$$

where $M_{1}=B_{1} B_{2} \ldots B_{k}\left(G_{k} B_{k}\right)^{-(k-1)} G_{k} \ldots G_{2}$ and $M_{2}=B_{2} \ldots B_{k}\left(G_{k} B_{k}\right)^{-(k-1)} G_{k} \ldots$ $G_{2} G_{1}$.

By the limit representation for the Drazin inverse proved in [11], we get the next limit representation for the CMP inverse.

Theorem 9. Let $A \in \mathbb{C}^{n \times n}$ with $\operatorname{ind}(A)=k$. If $k \leq l$, then

$$
A^{c, \dagger}=\lim _{\lambda \rightarrow 0} P_{R\left(A^{*}\right)} A^{l}\left(A^{l+1}+\lambda I\right)^{-1} P_{R(A)} .
$$

Proof. This expressions can be verified using the following limit representation for the Drazin inverse presented in [11]:

$$
A^{D}=\lim _{\lambda \rightarrow 0} A^{l}\left(A^{l+1}+\lambda I\right)^{-1} .
$$

Also, the corresponding limit representations of DMP and MPD inverses can be showed. 
Corollary 4. Let $A \in \mathbb{C}^{n \times n}$ with $\operatorname{ind}(A)=k$. If $k \leq l$, then

$$
A^{D, \dagger}=\lim _{\lambda \rightarrow 0} P_{R\left(A^{*}\right)} A^{l}\left(A^{l+1}+\lambda I\right)^{-1}
$$

and

$$
A^{\dagger, D}=\lim _{\lambda \rightarrow 0} A^{l}\left(A^{l+1}+\lambda I\right)^{-1} P_{R(A)} .
$$

As Theorem 4 and Theorem 5, we obtain some limit representations of CMP inverse which involve one limit.

Theorem 10. Let $A \in \mathbb{C}^{n \times n}$ with ind $(A)=k$. Then

$$
A^{c, \dagger}=\lim _{\lambda \rightarrow 0} A^{*}\left(\lambda I+A A^{*}\right)^{-1} P_{R\left(A^{k}\right), N\left(A^{k}\right)} P_{R(A)}=\lim _{\lambda \rightarrow 0} P_{R\left(A^{*}\right)} P_{R\left(A^{k}\right), N\left(A^{k}\right)} A^{*}\left(\lambda I+A A^{*}\right)^{-1} .
$$

For DMP and MPD inverses, the following limit representations hold.

Corollary 5. Let $A \in \mathbb{C}^{n \times n}$ with $\operatorname{ind}(A)=k$. Then

$$
A^{D, \dagger}=\lim _{\lambda \rightarrow 0} A^{*}\left(\lambda I+A A^{*}\right)^{-1} P_{R\left(A^{k}\right), N\left(A^{k}\right)}
$$

and

$$
A^{\dagger, D}=\lim _{\lambda \rightarrow 0} P_{R\left(A^{k}\right), N\left(A^{k}\right)} A^{*}\left(\lambda I+A A^{*}\right)^{-1} .
$$

We need one auxiliary result to prove new expressions for the CMP, DMP and MPD inverses.

Lemma 3 ([16]). Let $A \in \mathbb{C}^{m \times n}$ be of rank $r$, let $T$ be a subspace of $\mathbb{C}^{n}$ of dimension $s \leq r$, and let $S$ be a subspace of $\mathbb{C}^{m}$ of dimension $m-s$. In addition, suppose that $G \in \mathbb{C}^{n \times m}$ satisfies $R(G)=T$ and $N(G)=S$. If $A_{T, S}^{(2)}$ exists, then it possesses the limit representations

$$
A_{T, S}^{(2)}=\lim _{\lambda \rightarrow 0}(G A+\lambda I)^{-1} G=\lim _{\lambda \rightarrow 0} G(A G+\lambda I)^{-1} .
$$

Theorem 11. Let $A \in \mathbb{C}^{n \times n}$ with $\operatorname{ind}(A)=k$. If $G \in \mathbb{C}^{n \times n}$ such that $R(G)=$ $R\left(A^{\dagger} A^{k}\right)$ and $N(G)=N\left(A^{k} A^{\dagger}\right)$, then

$$
A^{c, \dagger}=\lim _{\lambda \rightarrow 0}(G A+\lambda I)^{-1} G=\lim _{\lambda \rightarrow 0} G(A G+\lambda I)^{-1} .
$$

Proof. By Lemma 3 (or [2, Corollary 7.5]), we have

$$
A^{c, \dagger}=A_{R\left(A^{\dagger} A^{k}\right), N\left(A^{k} A^{\dagger}\right)}^{(2)}=\lim _{\lambda \rightarrow 0}(G A+\lambda I)^{-1} G=\lim _{\lambda \rightarrow 0} G(A G+\lambda I)^{-1} .
$$

Theorem 12. Let $A \in \mathbb{C}^{n \times n}$ be of rank $r$ and $\operatorname{ind}(A)=k, B \in \mathbb{C}_{s}^{n \times s}$ and $C \in \mathbb{C}_{s}^{s \times n}$.

(i) Suppose that $R(B)=R\left(A^{\dagger} A^{k}\right)$ is a subspace of $\mathbb{C}^{n}$ of dimension $s \leq r$ and $N(C)=N\left(A^{k} A^{\dagger}\right)$ is a subspace of $\mathbb{C}^{n}$ of dimension $n-s$. Then

$$
A^{c, \dagger}=\lim _{t \rightarrow 0} B(t I+C A B)^{-1} C .
$$


(ii) Suppose that $R\left(B_{1}\right)=R\left(A^{k}\right)$ is a subspace of $\mathbb{C}^{n}$ of dimension $s \leq r$ and $N\left(C_{1}\right)=N\left(A^{k} A^{\dagger}\right)$ is a subspace of $\mathbb{C}^{n}$ of dimension $n-s$. If $\left(C_{1}\right)_{R\left(A B_{1}\right), N\left(A B_{1}\right)}^{(2)}$ exists, then

$$
A^{D, \dagger}=\lim _{t \rightarrow 0} B_{1}\left(t I+C_{1} A B_{1}\right)^{-1} C_{1}
$$

and

$$
A^{c, \dagger}=A^{\dagger}\left(C_{1}\right)_{R\left(A B_{1}\right), N\left(A B_{1}\right)}^{(2)} C_{1} .
$$

(iii) Suppose that $R\left(B_{2}\right)=R\left(A^{\dagger} A^{k}\right)$ is a subspace of $\mathbb{C}^{n}$ of dimension $s \leq r$ and $N\left(C_{2}\right)=N\left(A^{k}\right)$ is a subspace of $\mathbb{C}^{n}$ of dimension $n-s$. If $\left(B_{2}\right)_{R\left(C_{2} A\right), N\left(C_{2} A\right)}^{(2)}$ exists, then

$$
A^{\dagger, D}=\lim _{t \rightarrow 0} B_{2}\left(t I+C_{2} A B_{2}\right)^{-1} C_{2}
$$

and

$$
A^{c, \dagger}=B_{2}\left(B_{2}\right)_{R\left(C_{2} A\right), N\left(C_{2} A\right)}^{(2)} A^{\dagger} .
$$

Proof. (i) Applying [8, Theorem 7], we have that

$$
A_{R\left(A^{\dagger} A^{k}\right), N\left(A^{k} A^{\dagger}\right)}^{(2)}=\lim _{t \rightarrow 0} B(t I+C A B)^{-1} C .
$$

(ii) We firstly observe that $A^{D, \dagger}=A_{R\left(A^{k}\right), N\left(A^{k} A^{\dagger}\right)}^{(2)}$ and then, by [8, Theorem 7],

$$
A^{D, \dagger}=\lim _{t \rightarrow 0} B_{1}\left(t I+C_{1} A B_{1}\right)^{-1} C_{1} .
$$

Therefore, by Lemma 3 ,

$$
\begin{aligned}
A^{c, \dagger} & =A^{\dagger} A A^{D, \dagger}=A^{\dagger} \lim _{t \rightarrow 0} A B_{1}\left(t I+C_{1} A B_{1}\right)^{-1} C_{1} \\
& =A^{\dagger}\left(C_{1}\right)_{R\left(A B_{1}\right), N\left(A B_{1}\right)}^{(2)} C_{1} .
\end{aligned}
$$

(iii) This part can be proved in an analogy way as part (ii).

\section{REFERENCES}

[1] A. Ben-Israel and T. N. E. Greville, Generalized inverses: theory and applications. New Jork: Second Ed., Springer, 2003.

[2] J. Benítez, E. Boasso, and H. Jin, "On one-sided $(B, C)$-inverses of arbitrary matrices.” Electronic J. Linear Algebra, vol. 32, pp. 391-422, 2017, doi: 10.13001/1081-3810.3487.

[3] S. L. Campbell and C. D. Meyer, Generalized Inverses of Linear Transformations. London: Pitman, 1979.

[4] N. Castro-González, J. J. Koliha, and Y. Wei, "Integral representation of the Drazin inverse $A^{D}$," Electron. J. Linear Algebra, vol. 9, pp. 129-131, 2002.

[5] N. Castro-González, J. J. Koliha, and Y. Wei, "On integral representations of the Drazin inverse in Banach algebras." Proc. Edinb. Math. Soc., vol. 45, pp. 327-331, 2002, doi: 10.1017/S0013091500000523.

[6] M. P. Drazin, “A class of outer generalized inverses." Linear Algebra Appl., vol. 436, pp. 1909_ 1923, 2012, doi: 10.1016/j.laa.2011.09.004. 
[7] C. W. Groetsch, Generalized inverses of linear operators: representation and approximation, in: Monographs and Textbooks in Pure and Applied Mathematics, Vol. 37. New York, Basel: Marcel Dekker, Inc., 1977.

[8] X. Liu, Y. Yu, J. Zhong, and Y. Wei, "Integral and limit representations of the outer inverse in Banach space. ," Linear Multilinear Algebra, vol. 60, pp. 333-347, 2012, doi: 10.1080/03081087.2011.598154.

[9] S. B. Malik and N. Thome, "On a new generalized inverse for matrices of an arbitrary index." Appl. Math. Comput., vol. 226, pp. 575-580, 2014, doi: 10.1016/j.amc.2013.10.060.

[10] M. Mehdipour and A. Salemi, "On a new generalized inverse of matrices." Linear Multilinear Algebra, vol. 66, no. 5, pp. 1046-1053, 2018, doi: 10.1080/03081087.2017.1336200.

[11] C. D. Meyer, "Limits and the index of a square matrix." SIAM J. Appl. Math., vol. 26, pp. 469-478, 1974.

[12] D. Mosić, “The CMP inverse for rectangular matrices.” Aequationes Math., vol. 92, no. 4, pp. 649-659, 2018, doi: 10.1007/s00010-018-0570-7.

[13] D. Mosić and D. S. Djordjević, "The gDMP inverse of Hilbert space operators." Journal of Spectral Theory, vol. 8, no. 2, pp. 555-573, 2018, doi: 10.4171/JST/207.

[14] D. Mosić and M. Z. Kolundžija, "Weighted CMP inverse of an operator between Hilbert spaces." RACSAM, vol. 113, pp. 2155-2173, 2019, doi: 10.1007/s13398-018-0603-z.

[15] P. S. Stanimirović, "Limit representations of generalized inverses and related methods." Appl. Math. Comput., vol. 103, pp. 51-68, 1999, doi: 10.1016/S0096-3003(98)10048-6.

[16] Y. Wei, "A characterization and representation of the generalized inverse $A_{T, S}^{(2)}$ and its applications." Linear Algebra Appl., vol. 280, pp. 79-86, 1998, doi: 10.1016/S0024-3795(98)00008-1.

[17] Y. Wei and D. S. Djordjević, "On integral representation of the generalized inverse $A_{T, S}^{(2)}$ " Appl. Math. Comput., vol. 142, no. 1, pp. 189-194, 2003, doi: 10.1016/S0096-3003(02)00296-5.

[18] S. Xu, J. Chen, and D. Mosić, "New characterizations of the CMP inverse of matrices." Linear Multilinear Algebra, vol. 68, no. 4, pp. 790-804, 2020, doi: 10.1080/03081087.2018.1518401.

[19] A. Yu and C. Deng, "Characterizations of DMP inverse in a Hilbert space." Calcolo, vol. 51, no. 3, pp. 331-341, 2016, doi: 10.1007/s10092-015-0151-2.

[20] M. Zhou and J. Chen, "Integral representations of two generalized core inverses." Appl. Math. Comput., vol. 333, pp. 187-193, 2018, doi: 10.1016/j.amc.2018.03.085.

Author's address

Dijana Mosić

University of Niš, Faculty of Sciences and Mathematics, P.O. Box 224, 18000 Niš, Serbia

E-mail address: dijana@pmf.ni.ac.rs 